\title{
CARACTERIZAÇÃO DOS NÍVEIS DE ELEMENTOS QUÍMICOS EM SOLO, SUBMETIDO A DIFERENTES SISTEMAS DE USO E MANEJO, UTILIZANDO ESPECTROMETRIA DE FLUORESCENCIA DE RAIOS-X POR ENERGIA DISPERSIVA (EDXRF)
}

\author{
Arci Dirceu Wastowski*, Genesio Mario da Rosa, Maurício Roberto Cherubin e João Paulo Gonsiorkiewicz Rigon \\ Centro de Educação Superior Norte do Rio Grande do Sul, Universidade Federal de Santa Maria, Linha 7 de Setembro, s/n, \\ Br 386, km 40, 98400-000 Frederico Westphalen - RS, Brasil
}

Recebido em 18/8/09; aceito em 13/3/10; publicado na web em 20/7/10

\begin{abstract}
CHARACTERIZATION OF CHEMICAL ELEMENTS IN SOIL SUBMITTED TO DIFFERENT SYSTEMS USE AND MANAGEMENT BY ENERGY DISPERSIVE X-RAY FLUORESCENCE SPECTROMETRY (EDXRF). This study aimed to evaluate the chemical elements levels in soil, submitted to different management systems and use by the Energy Dispersive X-Ray Fluorescence Spectrometry - EDXRF. The systems were T1 - agro forestry (SAF), T2 - Native Field (CN), T3 - Native Forest (NM), T4 - Tillage Forest (PF); T5 - conventional tillage system (SPC) and T6 - System tillage (NT). Samples were collected at 0-10 and 10$20 \mathrm{~cm}$, dried and ground for analysis in EDX-720. The soil showed no difference in the average concentrations of chemical elements analyzed in the profiles, but the systems presented different concentrations of metal elements, and T3 had the highest $\mathrm{K}, \mathrm{Ca}$ and $\mathrm{Zn}$ at 0-10 $\mathrm{cm}$ and higher contents of $\mathrm{K}, \mathrm{Ca}, \mathrm{Cu}, \mathrm{Zn}$ and $\mathrm{Mn}$ in the layer of $10-20 \mathrm{~cm}$.
\end{abstract}

Keywords: metals in soil; chemical elements; energy dispersive X-ray fluorescence spectrometry.

\section{INTRODUÇÃO}

Nos últimos anos, tem-se intensificado a necessidade da adoção de sistemas de uso e manejo do solo que preservem ou promovam melhorias em suas características físicas, químicas e biológicas. Isto se deve ao aumento da população mundial e, em decorrência, à maior demanda por alimentos, gerando a necessidade de maior produtividade nas áreas agricultáveis. ${ }^{1}$ Desta forma, intensifica-se a importância de escolher sistemas mais adequados de uso e manejo do solo, que contribuam para a melhoria de suas qualidades agronômicas, assim mantendo ou alterando ao mínimo suas características e propriedades. . $^{2,3}$

A fertilidade do solo é um dos fatores que mais pode sofrer modificações, em função do uso e manejo do solo. Rachwal et al. ${ }^{4}$ destacam que a maioria dos solos do Brasil é pobre em nutrientes, porém as matas nativas não apresentam sintomas de deficiência nutricionais, em virtude da ciclagem dos nutrientes, estando esses em perfeito equilíbrio com as demandas.

Em relação aos sistemas agrícolas, são muitos os estudos que demonstram modificações químicas no solo entre eles destaca-se o de Muzilli, ${ }^{5}$ que encontrou maior acúmulo de nutrientes nas camadas superficiais em plantio direto, quando comparado ao convencional. Silva e Silveira ${ }^{1}$ apontam que os sistemas agrícolas afetam a distribuição de nutrientes no perfil do solo, ocorrendo maior concentração nas camadas superficiais naqueles em que houve menor movimentação de solo. Em sistemas florestais, Rachwal et al. destacam que a substituição de florestas naturais por plantios florestais de ciclo rápido altera os processos naturais de ciclagem e armazenamento de nutrientes, em razão, sobretudo, das mudanças na qualidade da matéria orgânica, causadas pelas práticas de manejo. ${ }^{4}$

Cientes das possíveis modificações nos níveis de nutrientes que um solo sob diferentes uso e manejo pode apresentar, e que o conhecimento da fertilidade dos solos cultivados é um dos fatores primordiais para a obtenção de sucesso na atividade agrícola, diversos autores ${ }^{6-9}$ buscaram caracterizar os níveis dos nutrientes encontrados em solos

\footnotetext{
*e-mail: wastowski@smail.ufsm.br
}

utilizados das mais variadas formas, por meio de amostragem de solo e análise química tradicional, conforme Tedesco et al.. ${ }^{10}$

Visando dinamizar este processo por meio de análises mais rápidas e de simples realização, a espectrometria de fluorescência de raios-X por energia dispersiva - EDXRF destaca-se como uma importante alternativa, com crescente aplicação na identificação mineralógica de solos, ${ }^{11-14}$ rochas, ${ }^{15,16}$ cerâmicas ${ }^{17}$ e líquidos, ${ }^{18}$ uma vez que permite a determinação simultânea ou sequencial da concentração de todos os elementos enquadrados entre o sódio $(\mathrm{Na})$ e o urânio (U), sem a necessidade de destruição da amostra, ou seja, de modo instrumental, sem nenhum pré-tratamento químico e podendo atingir limites de detecção da ordem de 1 a 20 ppm. ${ }^{19,20}$ Desta forma, objetivou-se com este trabalho caracterizar as alterações nos níveis dos metais, mais importantes para a nutrição de plantas, presentes num mesmo tipo de solo submetido a diferentes sistemas de uso e manejos, através da técnica de EDXRF.

\section{PARTE EXPERIMENTAL}

\section{Material e métodos}

O trabalho foi realizado no Centro de Educação Superior Norte do RS, da UFSM, cujas coordenadas são $27^{\circ} 23^{\prime} 47.58^{\prime \prime}$ S e $53^{\circ} 25^{\prime}$ 41.24" W, localizado em Frederico Westphalen - RS. O solo das áreas de estudo é um Latossolo Vermelho, ${ }^{21}$ segundo a classificação de Köppen, o clima da região é do tipo Cfa, com temperatura média anual em torno de $18{ }^{\circ} \mathrm{C}$, com máximas no verão podendo atingir $41{ }^{\circ} \mathrm{C}$ e mínimas no inverno atingindo valores inferiores a $0{ }^{\circ} \mathrm{C}$. A precipitação média anual é elevada, geralmente entre 1.800 e 2.100 $\mathrm{mm}$, bem distribuídos ao longo do ano. ${ }^{22}$

Foram coletadas amostras de solos em áreas próximas ao campus, compondo assim os tratamentos com diferentes sistemas de uso e manejo do solo: T1 - Sistema Agroflorestal (SAF) onde as espécies consorciadas são Pinus taeda e Ilex paraguaiensis e o solo é recoberto por grama; T2 - Campo Nativo $(\mathrm{CN})$ apresentando vegetação de gramíneas nativa da região; T3 - Mata Nativa (MN) fragmento de mata nativa, pertencente à região fitoecológica da Floresta Esta- 
cional Decidual, ${ }^{23} \mathrm{~T} 4$ - Plantio Florestal (PF): plantio de eucalipto (Eucalyptus spp.) com aproximadamente 25 anos de idade; T5 - Sistema de Plantio Convencional (SPC) com cultivo de milho no verão e pastagem no inverno; e T6 - Sistema de Plantio Direto (SPD) área com plantio direto consolidado a mais de 10 anos.

Em cada área foram coletadas 3 amostra de solo, sendo que cada uma é composta por 5 subamostras, coletadas de maneira que as mesmas fossem representativas do sistema de uso e manejo realizado na área, em 2 profundidades $(0-10$ e $10-20 \mathrm{~cm})$. Depois de coletadas, as amostras de solo foram secas em estufa de circulação forçada de ar sob temperatura de $105^{\circ} \mathrm{C}$ por $48 \mathrm{~h}$ e moídas em cadinho de porcelana. Para as análises posteriores no laboratório, das amostras secas e moídas, foi utilizada a fração da amostra de solo que passou pela peneira de 200 mesh.

As análises dos elementos químicos e suas quantidades presentes no solo foram realizadas no Laboratório de Análise e Pesquisas Químicas (LAPAQ - CESNORS/UFSM), por meio de um espectrômetro de fluorescência de raios-X por energia dispersiva, do modelo Shimadzu EDX-720. As seguintes condições de operação do equipamento foram selecionadas: tensão do tubo de $15 \mathrm{keV}$ (Na a Sc) e $50 \mathrm{keV}$ (Ti a U) com corrente no tubo de 184 e $25 \mu \mathrm{A}$, respectivamente; colimador de $10 \mathrm{~mm}$; tempo real de integração de $300 \mathrm{~s}$; tempo morto do detector de 40 e $39 \%$, sob vácuo e detector de $\mathrm{Si}(\mathrm{Li})$ refrigerado com nitrogênio líquido. O método analítico usado é denominado método dos parâmetros fundamentais (PF). ${ }^{17}$ Este método permite a obtenção da curva de sensibilidade do equipamento para cada elemento de interesse, quando uma amostra de composição química conhecida é submetida a parâmetros instrumentais bem definidos. A curva de sensibilidade do equipamento relaciona a intensidade fluorescente teórica calculada e a medida para cada elemento. ${ }^{17}$

Em geral, análise quantitativa por EDXRF é realizada pelo método da curva de calibração, obtida com muitos padrões. No entanto, para algumas aplicações é difícil obter padrões certificados suficientes, com matrizes semelhantes às amostras e, dessa forma, conseguir uma boa distribuição de pontos de dados sobre a escala de cada elemento a ser determinado. ${ }^{24-26}$ Han et al., a partir dos resultados da análise experimental de várias e diferentes amostras, comprovaram que resultados de alta precisão podem ser obtidos pelo método de PF, mesmo que apenas amostras de elemento puro sejam utilizadas para calibração. Isso também ilustra claramente que o método FP pode corrigir efetivamente o complicado cálculo do efeito da matriz. Portanto, para análise de rotina em massa, se as amostras de calibração estão ausentes, o método FP pode fornecer resultados relativamente precisos e quantitativos. ${ }^{27}$

A sensibilidade elementar é geralmente determinada pela medição das características dos raios-X emitidos por padrões, que contêm apenas um elemento. A utilização de padrões preparados em laboratório comercialmente disponíveis de elementos puros ou compostos tem se mostrado eficiente para a determinação da sensibilidade elementar em sistemas de XRF, porque são baratos e podem ser facilmente preparados. ${ }^{28}$ Para corrigir os efeitos de absorção e calibrar as linhas espectrais dos elementos analisados, utilizou-se o padrão A-750. O padrão de calibração utilizado, fornecido junto com o equipamento EDX-720, é uma liga metálica composta por alumínio, estanho, magnésio, ferro e cobre. Desta forma, é possível determinar a composição do material analisado.

Para as análises foram utilizadas aproximadamente $3 \mathrm{~g}$ de solo, acondicionadas sob um filme de Mylar ${ }^{\circledR}$ de $6 \mu \mathrm{m}$ de espessura, esticado no fundo de uma cela de polietileno com $32 \mathrm{~mm}$ de diâmetro externo e $23 \mathrm{~mm}$ de altura. Foram quantificados todos os elementos presentes no solo e avaliados os teores dos 9 principais metais presentes no solo sob o ponto de vista de nutrição de plantas, sendo estes: potássio $(\mathrm{K})$, fósforo $(\mathrm{P})$, cálcio $(\mathrm{Ca})$, enxofre $(\mathrm{S})$, alumínio $(\mathrm{Al})$, cobre $(\mathrm{Cu})$, zinco $(\mathrm{Zn})$, manganês $(\mathrm{Mn})$ e ferro $(\mathrm{Fe})$.

O delineamento experimental utilizado foi o inteiramente casualizado (DIC) com 3 repetições. Os resultados obtidos foram submetidos à análise de variância e as médias foram comparadas pelo teste de Tukey a $1 \%$ de probabilidade de erro.

\section{RESULTADOS E DISCUSSÃO}

$\mathrm{Na}$ caracterização dos teores de elementos químicos do solo através do EDXFR, verificou-se a presença de 16 elementos (Figura 1), nos sistemas amostrados e em ambas as profundidades, sendo este: silício ( $\mathrm{Si})$, ferro $(\mathrm{Fe})$, alumínio $(\mathrm{Al})$, titânio $(\mathrm{Ti})$, enxofre $(\mathrm{S})$, cálcio $(\mathrm{Ca})$, manganês $(\mathrm{Mn})$, vanádio $(\mathrm{V})$, potássio $(\mathrm{K})$, fósforo $(\mathrm{P})$, zircônio $(\mathrm{Zr})$, cobre $(\mathrm{Cu})$, cromo $(\mathrm{Cr})$, zinco $(\mathrm{Zn})$, nióbio $(\mathrm{Nb})$ e ítrio $(\mathrm{Y})$, respectivamente, em ordem decrescente de quantidade $\left(\mathrm{g} \mathrm{kg}^{-1}\right)$ presente no solo. Estes resultados, de modo geral, condizem com os encontrados por Rocha et al.. ${ }^{12}$

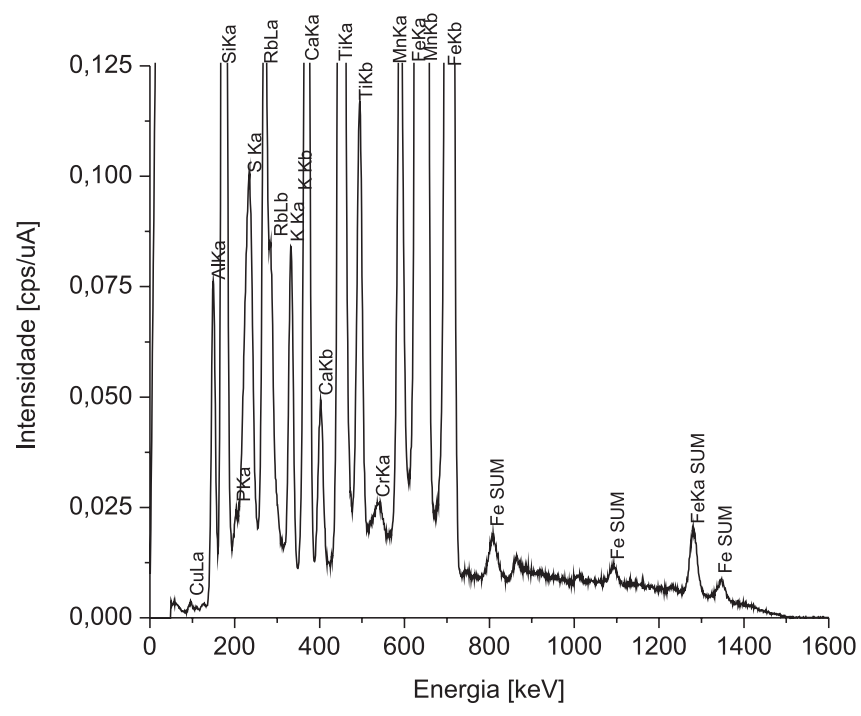

Figura 1. Espectro típico de uma amostra contendo todos os elementos encontrados no solo

Quanto aos valores de Ca (Tabela 1) se verificou que não há diferença entre os teores nas duas profundidades (0-10 e 10-20 cm) amostradas. Para os sistemas de manejo e uso, houve uma significativa variação (Figura 2), onde na camada superficial $(0-10 \mathrm{~cm})$ os maiores teores apresentados são do T3 e T6, respectivamente; pode-se, assim, presumir que são sistemas que provavelmente apresentam maiores teores de matéria orgânica, devido ao acúmulo de material orgânico na superfície do solo, oriundo principalmente das árvores caducifólias na floresta e dos restos culturais (palhada) no sistema de plantio direto, acarretando maior retenção do $\mathrm{Ca}^{2+}$ trocável nas superfícies negativas das argilas e da matéria orgânica. ${ }^{32}$

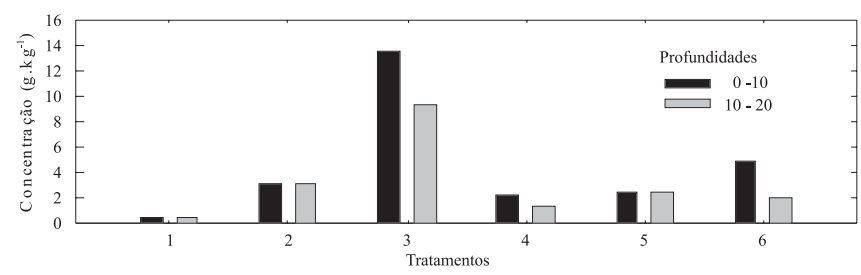

Figura 2. Concentração de cálcio (Ca) nos diferentes sistemas de uso e manejo do solo, nas profundidades de $0-10$ e $10-20 \mathrm{~cm}$

Além disso, no T6 são realizadas calagens regulares de acordo com resultados das análises de solo, caracterizando-se também como uma fonte de $\mathrm{Ca}$ ao solo. Estes resultados concordam com Sampaio $^{33}$ e Silva e Silveira, ${ }^{1}$ que também constataram maior con- 
Tabela 1. Teores de potássio $(\mathrm{K})$, fósforo $(\mathrm{P})$, cálcio $(\mathrm{Ca})$, enxofre $(\mathrm{S})$ e alumínio $(\mathrm{Al})$ presentes nos solos, obtidos através de espectrometria de fluorescência de raios-X por energia dispersiva e coeficiente de variação relativo (CV\%) das análises

\begin{tabular}{|c|c|c|c|c|c|c|}
\hline \multirow{2}{*}{$\begin{array}{l}\text { Profundidade } \\
---\mathrm{cm}---\end{array}$} & \multirow{2}{*}{ Trat. } & \multicolumn{5}{|c|}{ Elementos $\left(\mathrm{g} \mathrm{kg}^{-1}\right)$} \\
\hline & & $\mathrm{K}$ & $\mathrm{P}$ & $\mathrm{Ca}$ & $\mathrm{S}$ & $\mathrm{Al}$ \\
\hline & 1 & $0,78 \mathrm{~d}^{*}$ & $0,81 \mathrm{a}$ & $0,53 \mathrm{e}$ & $5,43 \mathrm{a}$ & $108,27 \mathrm{a}$ \\
\hline & 2 & $1,76 \mathrm{~b}$ & $0,75 \mathrm{a}$ & $3,09 \mathrm{c}$ & $5,08 \mathrm{a}$ & $94,67 \mathrm{~b}$ \\
\hline \multirow[t]{5}{*}{$0-10$} & 3 & $4,92 \mathrm{a}$ & $1,08 \mathrm{a}$ & $13,68 \mathrm{a}$ & $5,59 \mathrm{a}$ & $69,13 \mathrm{~d}$ \\
\hline & 4 & $1,26 \mathrm{c}$ & $0,68 \mathrm{a}$ & $2,13 \mathrm{~d}$ & $5,00 \mathrm{a}$ & $70,92 \mathrm{~d}$ \\
\hline & 5 & $1,06 \mathrm{c}$ & $0,79 \mathrm{a}$ & $2,37 \mathrm{~d}$ & $3,92 \mathrm{a}$ & $79,01 \mathrm{c}$ \\
\hline & 6 & $1,23 \mathrm{c}$ & $1,31 \mathrm{a}$ & $4,91 \mathrm{~b}$ & $5,23 \mathrm{a}$ & $92,21 \mathrm{~b}$ \\
\hline & Média & $1,84 \mathrm{~A}$ & $0,90 \mathrm{~A}$ & $4,45 \mathrm{~A}$ & $5,04 \mathrm{~A}$ & $85,70 \mathrm{~A}$ \\
\hline \multirow[t]{3}{*}{ CV\%** Sistema } & & 4,28 & 48,79 & 4,1 & 2,34 & 2,34 \\
\hline & 1 & $0,72 \mathrm{~d}$ & $0,86 \mathrm{a}$ & $0,38 \mathrm{e}$ & $5,44 \mathrm{a}$ & $112,38 \mathrm{a}$ \\
\hline & 2 & $2,03 \mathrm{~b}$ & $0,92 \mathrm{a}$ & $3,16 \mathrm{~b}$ & $5,17 \mathrm{a}$ & $94,59 \mathrm{~b}$ \\
\hline \multirow[t]{5}{*}{$10-20$} & 3 & $4,59 \mathrm{a}$ & $0,54 \mathrm{a}$ & $9,44 \mathrm{a}$ & $5,16 \mathrm{a}$ & $79,65 \mathrm{c}$ \\
\hline & 4 & $1,11 \mathrm{c}$ & $1,32 \mathrm{a}$ & $1,35 \mathrm{~d}$ & $6,41 \mathrm{a}$ & $75,39 \mathrm{c}$ \\
\hline & 5 & $1,01 \mathrm{~cd}$ & $0,52 \mathrm{a}$ & $2,38 \mathrm{c}$ & $4,51 \mathrm{a}$ & $88,99 \mathrm{~b}$ \\
\hline & 6 & $0,78 \mathrm{~d}$ & $0,86 \mathrm{a}$ & $1,88 \mathrm{c}$ & $4,88 \mathrm{a}$ & $72,76 \mathrm{c}$ \\
\hline & Média & $1,71 \mathrm{~A}$ & $0,84 \mathrm{~A}$ & $3,10 \mathrm{~A}$ & $5,26 \mathrm{~A}$ & $87,29 \mathrm{~A}$ \\
\hline CV\% Sistema & & 6,24 & 40,23 & 6,16 & 13,3 & 3,53 \\
\hline CV\% Profundidade & & 85,69 & 30,87 & 107,6 & 12,12 & 17,41 \\
\hline
\end{tabular}

*As médias seguidas pela mesma letra minúscula na coluna não diferem estatisticamente entre si nos tratamentos, e as médias seguidas por mesmas letras maiúsculas na coluna não diferem estatisticamente entre si nas profundidades, pelo teste de Tukey ao nível de $1 \%$ de probabilidade. ** Coeficiente de variação.

centração de Ca no sistema de plantio direto quando comparados a outros sistemas agrícolas.

O T1 e T4 apresentaram os menores teores do Ca, podendo ser explicado pela considerável extração do nutriente existente nestes sistemas, pelas espécies de rápido crescimento, como o Eucalyptus e o Pinus..$^{34,35}$ Além disso, no T1 a acidificação do solo promovida pelas acículas de Pinus provoca uma redução dos teores de cálcio. ${ }^{32}$

Os teores de P (Tabela 1) não apresentaram diferença significativa entre as profundidades no solo; também não se observou diferença significativa entre os sistemas de manejo, porém cabe uma ressalva em relação ao coeficiente de variação relativo $(\mathrm{CV} \%)$ encontrado. Este coeficiente mensura a dispersão dos valores em relação à média, assim, observou-se que em relação aos outros elementos os valores do CV\% do fósforo, com 48,79 e 40,23\%, respectivamente, nas profundidades de $0-10$ e 10-20 cm, foram considerados expressivos. Silva e Silveira ${ }^{1}$ ao compararem os sistemas agrícolas, considerando cada camada de solo quanto ao teor de fósforo extraível, também não constataram diferença significativa para os perfis que analisaram, contudo, ressaltaram os elevados CVs\%, chegando a 104,59\%, o que comprometeu a interpretação desses dados. Souza et al., ${ }^{33} \mathrm{em}$ estudo com solos sob diferentes manejos, também encontraram altos valores de coeficientes de variação para $\mathrm{P}$ e $\mathrm{K}$, chegando a atingir mais de $100 \%$.

As concentrações de $\mathrm{K}$ não diferiam significativamente entre as profundidades, sendo que os maiores teores foram encontrados no T3 (Tabela 1), podendo ser esta atribuída ao fato que neste sistema há uma maior fonte orgânica de $\mathrm{K}$, em virtude da maior deposição de material orgânico no solo. Lopes et al., ${ }^{7}$ em estudo de propriedades químicas de um gleissolo sob diferentes sistemas de uso, também verificaram que as maiores concentrações de potássio se encontram sob mata nativa. As menores concentrações foram encontradas em
T1; este resultado pode ser atribuído ao fato do sistema proporcionar uma grande exportação do elemento pelas plantas de erva-mate e pelos Pinus, considerando também que na área nunca foi realizada uma adubação química potássica.

Os teores de $\mathrm{S}$ não diferiram entre os sistemas de uso e manejo em ambas as profundidades estudadas (Tabela 1), o que demonstra que os sistemas não conseguiram alterar a quantidade deste elemento no solo.

Os teores de $\mathrm{Al}$ foram maiores no $\mathrm{T} 1$, em ambas as profundidades (Tabela 1); isso pode ser atribuído, segundo Souza e Souza, ${ }^{32}$ ao acúmulo de acículas de Pinus sobre o solo, neste sistema, o que acarreta na acidificação do solo e, consequentemente, na liberação do alumínio. Já os outros dois sistemas que possuem vegetação arbórea, T3 e T4, apresentaram os menores teores, não diferindo estatisticamente entre si.

Quanto aos teores de cobre (Tabela 2) se verificou que na camada mais superficial T6 e T5 apresentaram maiores concentrações; estes resultados podem ser explicados pelas adubações químicas e pela adição periódica de dejetos suínos, ricos em micronutrientes, realizadas ao longo dos anos de uso do solo. De acordo com Girotto et al., ${ }^{34}$ aplicação sistemática de altas doses de dejeto suíno no solo provoca acúmulo de $\mathrm{Cu}$ nas camadas superficiais do solo. Já na profundidade de 10-20 cm T3 também se destacou, provavelmente pelo maior teor de matéria orgânica $(\mathrm{MO})$ em profundidade, retendo fortemente o elemento. ${ }^{32} \mathrm{O}$ T5 e T6 não diferiram entre si, concordando com Oliveira et al..$^{35}$ e Silva e Silveira, ${ }^{1}$ que em avaliações de sistemas agrícolas também não encontraram diferenças significativas de $\mathrm{Cu}$ nas camadas superficiais. As concentrações de $\mathrm{Zn}$ não tiveram diferença em profundidade; já entre os sistemas estudados, T3 e T6 obtiveram os maiores teores, fato que deve estar ligado ao maior acúmulo de matéria orgânica presente em tais sistemas.

Em ambas as profundidades de solo avaliadas, os teores de $\mathrm{Mn}$ diferiram em função do sistema de uso e manejo, sendo que na camada de 0-10 cm, T2 e T3, respectivamente, apresentaram teores

Tabela 2. Teores de cobre $(\mathrm{Cu})$, Zinco $(\mathrm{Zn})$ e manganês $(\mathrm{Mn})$ presentes nos solos, obtidos através de espectrometria de fluorescência de raios-X por energia dispersiva e coeficiente de variação relativo (CV\%) das análises

\begin{tabular}{|c|c|c|c|c|c|}
\hline \multirow{2}{*}{$\begin{array}{l}\text { Profundidade } \\
---\mathrm{cm} \text {--- }\end{array}$} & \multirow{2}{*}{ Trat. } & \multicolumn{4}{|c|}{ Elementos $\left(\mathrm{g} \mathrm{kg}^{-1}\right)$} \\
\hline & & $\mathrm{Cu}$ & $\mathrm{Zn}$ & Mn & $\mathrm{Fe}$ \\
\hline \multirow{6}{*}{0 a 10} & 1 & $0,38 \mathrm{c}^{*}$ & $0,13 \mathrm{~b}$ & $1,81 \mathrm{~d}$ & $136,97 \mathrm{c}$ \\
\hline & 2 & $0,47 \mathrm{~b}$ & $0,19 a b$ & $4,97 \mathrm{a}$ & $158,83 \mathrm{~b}$ \\
\hline & 3 & $0,47 \mathrm{~b}$ & $0,23 \mathrm{a}$ & $4,20 \mathrm{~b}$ & $139,21 \mathrm{c}$ \\
\hline & 4 & $0,47 \mathrm{~b}$ & $0,17 \mathrm{ab}$ & $3,24 \mathrm{c}$ & $161,70 \mathrm{~b}$ \\
\hline & 5 & $0,51 \mathrm{ab}$ & $0,21 \mathrm{ab}$ & $3,74 \mathrm{bc}$ & $180,86 \mathrm{a}$ \\
\hline & 6 & $0,55 \mathrm{a}$ & $0,23 \mathrm{a}$ & $4,00 \mathrm{~b}$ & $190,35 \mathrm{a}$ \\
\hline \multirow{3}{*}{ CV\% Sistema } & Média & 0,47 A & $0,19 \mathrm{~A}$ & $3,66 \mathrm{~A}$ & $161,32 \mathrm{~A}$ \\
\hline & & 4,69 & 17,69 & 6,34 & 4,16 \\
\hline & 1 & $0,45 \mathrm{c}$ & $0,15 \mathrm{~d}$ & $2,10 \mathrm{e}$ & $156,66 \mathrm{bc}$ \\
\hline \multirow{6}{*}{10 a 20} & 2 & $0,43 \mathrm{c}$ & $0,17 \mathrm{~cd}$ & $2,93 \mathrm{~d}$ & $146,36 \mathrm{c}$ \\
\hline & 3 & $0,59 \mathrm{a}$ & $0,26 \mathrm{a}$ & $5,04 \mathrm{a}$ & $174,60 \mathrm{ab}$ \\
\hline & 4 & $0,47 \mathrm{bc}$ & $0,22 \mathrm{~b}$ & $3,03 \mathrm{~cd}$ & $165,60 \mathrm{bc}$ \\
\hline & 5 & $0,53 \mathrm{ab}$ & $0,21 \mathrm{~b}$ & $3,95 \mathrm{~b}$ & $192,26 \mathrm{a}$ \\
\hline & 6 & $0,47 \mathrm{bc}$ & $0,19 \mathrm{bc}$ & $3,42 \mathrm{c}$ & $161,64 \mathrm{bc}$ \\
\hline & Média & 0,49 A & $0,20 \mathrm{~A}$ & $3,41 \mathrm{~A}$ & $166,19 \mathrm{~A}$ \\
\hline CV\% Sistema & & 5,92 & 5,48 & 5,1 & 4,76 \\
\hline CV\% Profundidade & & 12 & 19,78 & 29,34 & 11,54 \\
\hline
\end{tabular}

*As médias seguidas pela mesma letra minúscula na coluna não diferem estatisticamente entre si nos tratamentos, e as médias seguidas por mesmas letras maiúsculas na coluna não diferem estatisticamente entre si nas profundidades, pelo teste de Tukey ao nível de $1 \%$ de probabilidade. 
elevados (Tabela 2). Já na profundidade de $10-20 \mathrm{~cm}$ os sistemas que com elevados teores foram T3 e T5. Em ambas as profundidades, o sistema T1 apresentou os menores valores deste mineral. Os teores de Fe encontrados foram os maiores entre os metais avaliados (Tabela 2), devendo estar relacionado ao tipo de material de origem do solo, rico em minerais com a hematita $\left(\mathrm{Fe}_{2} \mathrm{O}_{3}\right)$, olivina $\left((\mathrm{MgFe})_{2} \mathrm{SiO}_{4}\right.$, e goethita $(\mathrm{FeO}(\mathrm{OH}))$. Os teores médios de ferro não variaram em profundidade. Porém, entre os sistemas, os maiores teores na camada superficial foram encontrados nos dois sistemas agrícolas T5 e T6. Em estudo comparando somente solos com plantio direto e convencional, Castro et al. ${ }^{34}$ também não encontraram diferenças no teor de Fe, entre os sistemas.

\section{CONCLUSÃO}

Os resultados obtidos pela espectrometria de fluorescência de raios-X por energia dispersiva, EDXRF, podem ser utilizados para avaliação das modificações existentes nos teores de elementos químicos presentes no solo submetido a diferentes sistemas de uso e manejo.

O solo não apresentou diferença nas concentrações médias dos elementos químicos analisados nos perfis avaliados, porém os sistemas apresentam variação nas concentrações dos metais, sendo que T3 apresentou os maiores teores de K, Ca e Zn na camada de $0-10 \mathrm{~cm}$ e os maiores teores de $\mathrm{K}, \mathrm{Ca}, \mathrm{Cu}, \mathrm{Zn}$ e $\mathrm{Mn}$ na camada de $10-20 \mathrm{~cm}$.

Dentre os metais avaliados, o Fe apresentou as maiores concentrações no solo, em ambas as profundidades, indicando a presença de minerais ricos neste elemento na constituição do material de origem do solo.

\section{REFERÊNCIAS}

1. Silva, C. C.; Silveira, P. M.; Ciência Agrotécnica 2002, 26, 505.

2. Ribon, A. A.; Centurion, J. F.; Centurion, M. A. P. C.; Carvalho Filho, A.; Revista Brasileira de Ciência do Solo 2002, 26, 781.

3. Costa, F. S.; Albuquerque, J. A.; Bayer, C.; Fontoura, S. M. V.; Wobeto, C.; Revista Brasileira de Ciência do Solo 2003, 27, 527.

4. Rachwal, M. F. G.; Dedecek, R. A.; Curcio, G. R.; Simon, A. A.; Ciência Florestal 2007, 17, 137.

5. Muzilli, O.; Revista Brasileira de Ciência do Solo 1983, 7, 95.

6. Perin, E.; Ceretta, C. A.; Klamt, E.; Revista Brasileira de Ciência do Solo 2003, 27, 665 .

7. Lopes, E. L. N.; Fernandes, A. R.; Grimaldi, C.; Ruivo, M. L. P.; Rodrigues, T. E.; Sarrazin, M.; Boletim do Museu Paraesense Emílio Goeldi, Ciências Naturais 2006, 1, 127.

8. Mota, H.; Pequeno, P. L. L.; Brogio, M. P.; Shlindwein, J. A.; Resumo do Congresso Brasileiro de Ciência do solo, Gramado, Brasil, 2007.

9. Frazão, L. A.; Píccolo, M. C.; Feigl, B. J.; Cerri, C. C.; Cerri, C. E. P.; Pesquisa Agropecuária Brasileira 2008, 43, 641.

10. Tedesco, M. J.; Gianello, C.; Bissani, C. A.; Bohnen, H.; Volkweiss, S. J.; Análise de Solo, Plantas e Outros Materiais, 2a ed., UFRGS: Porto Alegre, 1995.
11. Dantas, C. C.; Dantas, H. S.; Dack, L. V.; Griekem, R. V.; Quim. Nova 1981, 4, 110.

12. Rocha, C. A. A.; Ferreira, J. R.; Mauri, J.; Alexandre, J.; Resumo do $51^{\circ}$ Congresso Brasileiro de Cerâmica, Salvador, Brasil, 2007.

13. Sitko, R.; Zawisza, B.; Jurczyk, J.; Buhll, F.; Zielonka, U.; Pol. J. Environ. Studies 2004, 13, 91.

14. Yu, K. N.; Yeung, Z. L. L; Lee, L. Y. L.; Stokes, M. J.; Kwok, R. C. W.; Appl. Radiat. Isot. 2002, 57, 279.

15. Albers, A. P. F.; Melchiades, F. G.; Machado, R.; Baldo, J. B.; Boschi, A. O.; Cerâmica 2002, 48, 34.

16. Ferreira, B. A.; Fabris, J. D.; Santana, D. P.; Curi, N.; Revista Brasileira de Ciência do Solo 2003, 27, 405.

17. Bona, I. A. T.; Sarkis, J. E. S.; Salvador, V. L. R.; Quim. Nova 2007, 30, 785.

18. Pataca, L. C. M.; Bortoleto, G. G.; Bueno, M. I. M. S.; Quim. Nova 2005, 28, 579 .

19. Nagata, N.; Bueno, M. I. M. S.; Peralta-Zamora, P. G.; Quim. Nova 2001, 24, 531.

20. Mamani, M. C. V.; Dissertação de Mestrado, Universidade Estadual de Campinas, Brasil, 2003.

21. Embrapa; Sistema Brasileiro de Classificação de Solos, Centro Nacional de Pesquisa de Solos: Rio de Janeiro, 1999.

22. Bernardi, I. P.; Pulchério-Leite, A.; Miranda, J. M. D.; Passos, F. C.; Revista Brasileira de Zoologia 2007, 24, 505.

23. IBGE; Manual Técnico da Vegetação Brasileira, Fundação Instituto Brasileiro de Geografia e Estatística: Rio de Janeiro, 1991.

24. Omote, J.; Kohno, H.; Toda, K.; Anal. Chim. Acta 1995, 307, 117.

25. Marguí, E.; Hidalgo, M.; Queralt, I.; Spectrochim. Acta, Part B 2005, 60, 1363.

26. Marguí, E.; Queralt, I.; Hidalgo, M.; Trends Anal. Chem. 2009, $28,362$.

27. Han, X. Y.; Zhuo, S. J.; Shen, R. X.; Wang, P. L.; Ji, A.; J. Quant. Spectrosc. Radiat. Transfer 2006, 97, 68.

28. Lopes, A. S.; Manual de Fertilidade do Solo, Editora Anda/Potafos: São Paulo, 1989.

29. Sampaio, G. V.; Dissertação de Mestrado, Universidade Federal de Viçosa, Brasil, 1987.

30. Castro, O. M.; Camargo, O. A.; Cantarella, H.; Veira, S. R.; Dechen, S. C. F.; Bragantia, Campinas 1992, 51, 77.

31. Gonçalves, J. L. M.; Circular Técnica $N^{\circ} 154$, IPEF - Instituto de Pesquisas e Estudos Florestais, Piracicaba, Brasil, 1988.

32. Souza, D. M. P.; Souza, M. L. P.; Revista Floresta 1981, 12, 36.

33. Souza, L. S.; Cogo, N. P.; Vieira, S. R.; Revista Brasileira de Ciência do Solo 1998, 22, 77.

34. Girotto, E.; Ceretta, C. A.; Bruneto, G.; Lourenzi, C. R.; Vieira, R. C. B.; Lorensini, F.; Trentin, E. E.; Resumos do XXI Congresso Brasileiro de Ciência do Solo, Gramado, Brasil, 2007.

35. Oliveira, I. P.; Kluthcouski, J.; Dos Santos, R. S. M.; Francelli, A. L.; Neto, D. D.; Faria, C. D.; Pesquisa Agropecuária Tropical 2001, 31, 97. 